\title{
Thermal sensitivity of carbon nanotube and graphene oxide containing responsive hydrogels
}

\author{
E. Manek ${ }^{1}$, B. Berke ${ }^{1,2}$, N. Miklósi ${ }^{1}$, M. Sajbán ${ }^{1}$, A. Domán ${ }^{1}$, T. Fukuda ${ }^{3}$, O. Czakkel ${ }^{2}$, K. László ${ }^{1 *}$ \\ ${ }^{1}$ Budapest University of Technology and Economics, Department of Physical Chemistry and Materials Science, \\ 1521 Budapest, Hungary \\ ${ }^{2}$ Institut Laue Langevin, CS 20156, F - 38042 Grenoble Cedex 9, France \\ ${ }^{3}$ Toyo University, Bio-Nano Research Centre, 350-8585 Kujirai, 2100 Kawagoe, Japan
}

Received 27 November 2015; accepted in revised form 20 March 2016

\begin{abstract}
Comparative investigations are reported on poly( $N$-isopropylacrylamide) (PNIPA) gels of various carbon nanotube (CNT) and graphene oxide (GO) contents synthesized under identical conditions. The kind and concentration of the incorporated carbon nanoparticles (CNPs) influence the swelling and stress-strain behaviour of the composites. Practically independently of the filler content, incorporation of CNPs appreciably improves the fracture stress properties of the gels. The time constant and the swelling ratio of the shrinkage following an abrupt increase in temperature of the swelling medium from 20 to $50^{\circ} \mathrm{C}$ can be adjusted by selecting both the type and the amount of nanoparticle loading. This offers a means of accurately controlling the deswelling kinetics of drug release with PNIPA systems, and could be employed in sensor applications where fast and excessive shrinkage are a significant drawback. Both CNTs and GO enhance the infrared sensitivity of the PNIPA gel, thus opening a route for the design of novel drug transport and actuator systems. It is proposed that the influence of the CNPs depends more on their surface reactivity during the gel synthesis rather than on their morphology. One of the important findings of this study is the existence of a thermally conducting network in the GO filled gels.
\end{abstract}

Keywords: polymer composites, nanocomposites, polymer gels, nanocarbon hybrids, thermal relaxation

\section{Introduction}

Smart hydrogels display a marked response under changes in certain external factors [1,2] such as composition of the solvent mixture [3], $\mathrm{pH}$ [4], temperature [5], photons, electric or magnetic fields [6]. Their softness, biocompatibility, excellent swelling properties and ability to deform reversibly, make them desirable as vehicles for controlled drug delivery [7, 8], sensors [9] or actuators [10]. Poly ( $N$-isopropylacrylamide) (PNIPA) based hydrogels are among the most studied temperature-responsive systems, as they exhibit a volume phase transition around $34^{\circ} \mathrm{C}$, close to the temperature of the (human) body. This property can be of use in biomedical applications $[11,12]$. These systems nevertheless have drawbacks, such as weak mechanical strength, which restricts applications involving repetitive loading. Composite hydrogels that incorporate nanoparticles in the matrix may offer a solution. Nanoparticles can either be physically trapped within the hydrogel matrix or cross-linked into the network structure by surface functionalities, resulting in nanocomposite materials that possess not only improved mechanical strength but also well-defined optical, thermal, electronic, magnetic, etc. properties [13].

The outstanding mechanical and conductive properties of carbon nanoparticles (CNPs), together with their unique structure and low density, place them among the most common fillers. Carbon nanofiber and carbon nanotubes (CNT) [14-20] as well as graphene 
and its derivatives [21-24], mainly graphene oxide (GO), have attracted marked interest in the last few years. In biomedical applications, however, the uncertainty surrounding the toxicity of CNTs [14] could affect choices of nanocarbon filler. GO, by contrast, is reported to be non-toxic and highly biocompatible [14].

In addition to mechanical reinforcement, the strong light absorption of nanocarbons can add new functional sensitivity to composite gels [25]. Fast reversible optical response has been observed under near infrared (NIR) laser excitation in CNT [26, 27] and GO [25] containing composite PNIPA hydrogels. NIR irradiation causes strong warming of the GO composite gels, while in hydrogels without GO no heating is observed [25].

CNP containing composite systems have been widely investigated in the recent years. In most cases, a synergetic effect is clearly observed upon incorporation of nanofillers into a polymer matrix, although in certain cases negative synergy has been reported. The preparation process and the type of carbon filler used determine the interactions between the filler particles as well as between the fillers and the polymer matrix [28]. Investigations regarding CNT [29-31] and GO [32-34] containing PNIPA based composite systems show improved mechanical properties (elastic modulus and compressive strength). As these gels were investigated with a variety of cross-link densities and/or with different co-monomers, as well as diverse conditions of synthesis, it is not possible to evaluate the effects of the different nanoparticles.

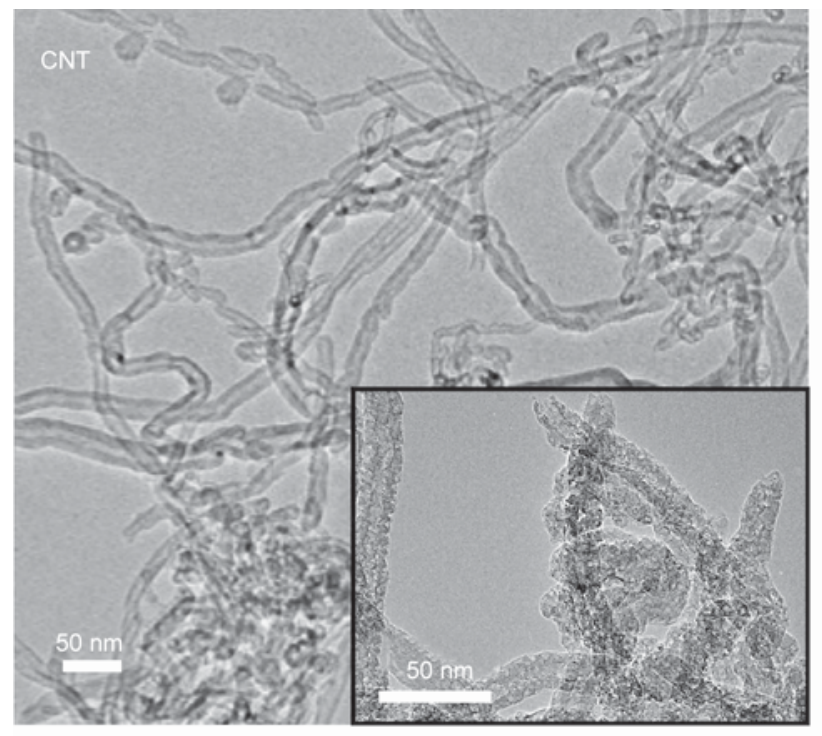

a)
In this paper we describe results from CNT and GO containing PNIPA gels synthesized under identical conditions. This allows a direct comparison of the macroscopic (swelling and stress-strain behaviour) and microscopic (scanning electron microscopic) properties of these systems. The application related performance of these composites is characterized by their infrared sensitivity and the kinetic response of their volume after an external temperature jump.

\section{Materials and methods}

\subsection{Carbon nanoparticles}

Typical images of the CNPs investigated are shown in Figure 1. The dispersibility of the CNTs in aqueous medium was improved by oxidizing commercial multi-wall CNT (external diameter: 10-20 nm; length: $10-30 \mu \mathrm{m}$ according to the supplier) produced by Chengdu Organic Chemicals Co. Ltd. (Sichuan, China) in concentrated $\mathrm{HNO}_{3}(65 \%$, Merck) (3 hours, $110^{\circ} \mathrm{C}$ ) in accordance with the procedure (including purification) of ref [35]. The oxidation process had practically no effect on the external diameter of the CNTs. The C/O ratio of the CNT (from X-ray photoelectron spectroscopy XPS) and the surface area $S_{\mathrm{BET}}$ (determined from low temperature nitrogen adsorption applying the Brunauer-Emmett-Teller model) were respectively 11.2 and $221 \mathrm{~m}^{2} / \mathrm{g}$.

Graphene oxide (GO) was obtained from natural graphite (from Madagascar) by the improved Hummer method [36]. The pristine GO suspension was purified by successive centrifugation (Jouan BR4i Multifunction Centrifuge, Thermo Scientific, USA;

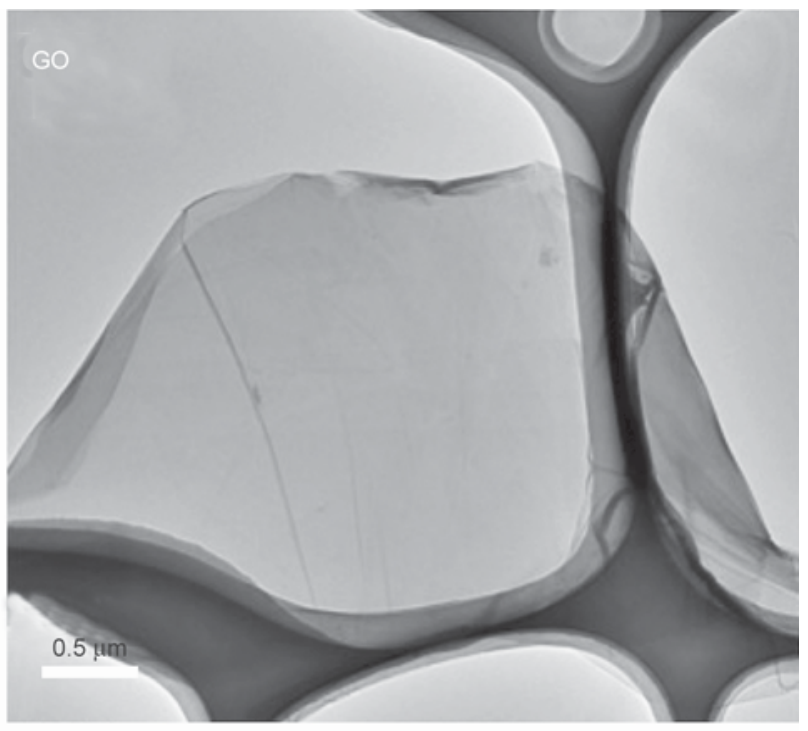

b)

Figure 1. High resolution transmission electron microscopy (HRTEM) images of oxidized CNT (a) and GO (b) 
$5000 \mathrm{~min}^{-1}$ ) and thorough washing with $1 \mathrm{M} \mathrm{HCl}$ and doubly distilled water. The stock suspension contained $0.2 \mathrm{w} / \mathrm{w} \% \mathrm{GO}$. The $\mathrm{C} / \mathrm{O}$ ratio and $S_{\mathrm{BET}}$ meast respectively.

\subsection{Gel synthesis}

The PNIPA polymer gel was synthesised from $N$-isopropylacrylamide (NIPA) monomer (Tokyo Chemical Industry Co., LTD., Tokyo, Japan) and $N, N^{\prime}-$ methylenebisacrylamide (BA) cross-linker (Sigma Aldrich) in aqueous medium with nominal molar ratio of $[\mathrm{NIPA}] /[\mathrm{BA}]=150$ at $20^{\circ} \mathrm{C}$ by free radical polymerization. The reaction was initiated by ammonium persulphate (APS, Sigma Aldrich) and $N, N, N^{\prime}, N^{\prime}$ tetramethylethylenediamine (TEMED, Fluka). A detailed description is given elsewhere [37]. All chemicals were used as received except NIPA, which was recrystallized from toluene-hexane mixture prior to the synthesis. $2 \mathrm{~mm}$ thick films and $10 \times 10 \mathrm{~mm}$ isometric cylinders were cast. Doubly distilled water was used in all experiments, including both synthesis and dialysis.

To obtain composite gels the aqueous CNP suspension of the required concentration was mixed with the precursor solution. For the CNT containing composites ultrasonication was used to ensure homogeneity of the dispersion. Gels with $\geq 20 \mathrm{mg} \mathrm{GO} / \mathrm{g}$ NIPA content were prepared by adding in succession solid NIPA and BA to the GO suspension. The reaction medium was stirred in an ice-bath for $15 \mathrm{~min}$ after addition of each component. The precursor suspensions were polymerised and purified in the same way as the nanoparticle-free PNIPA gel. No external field was applied. The films were stored in the swollen state for later use, unless mentioned otherwise.

\subsection{Scanning electron microscopy (SEM)}

Dialysed gel films fully swollen at room temperature were frozen in liquid nitrogen and broken immediately (Figure 2) prior to lyophilization (Scanvac Coolsafe freeze dryer, Lynge, Denmark; $T=25{ }^{\circ} \mathrm{C}, p=$ $10^{-2}$ mbar, 24 hours). After coating with metal alloy (atomic ratio $\mathrm{Au}: \mathrm{Pd}=1.5: 1$ ), SEM images were taken with a Hitachi SU6600 analytical variable pressure scanning electron microscope equipped with a $\mathrm{ZrO} / \mathrm{W}$ Schottky field emission electron source and an environmental secondary electron detector (Hitachi

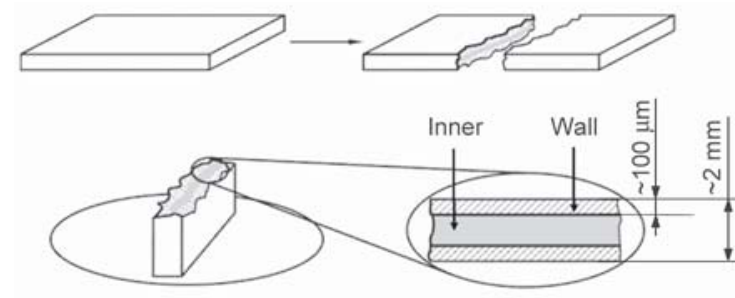

Figure 2. Preparation of gel films for SEM observation. The upper cartoon shows how the film was broken prior to freeze-drying. The lower cartoon shows how the sample was fixed on the SEM sample holder and gives the tentative thickness of the wall and bulk regions, respectively.

Ltd., Tokyo, Japan). The size distribution of the pores was determined from 80-100 data for each sample.

\subsection{Macroscopic characterisation}

To determine the equilibrium swelling degree $\mathrm{m} / \mathrm{m}_{0}$ (where $m$ and $m_{0}$ are the mass of the swollen and the dry gel sample, respectively), $7 \mathrm{~mm}$ diameter discs cut from the dialysed swollen film were dried to constant mass in a desiccator over concentrated $\mathrm{H}_{2} \mathrm{SO}_{4}$, and then re-swollen to equilibrium in excess water at $20.0 \pm 0.2{ }^{\circ} \mathrm{C}$.

Stress-strain studies were performed on fully swollen isometric $(10 \times 10 \mathrm{~mm})$ gel cylinders with an INSTRON 5543 (INSTRON, Norwood, USA) mechanical testing equipment at ambient temperature. Samples were compressed until fracture, in steps of $0.1 \mathrm{~mm}$ in the small deformation region $(<10 \%$ of their initial height) and in steps of $0.5 \mathrm{~mm}$ thereafter. The relaxation time and force threshold was $4 \times 4 \mathrm{~s}$ and $300 \mathrm{~N}$, respectively. The elastic modulus was determined by the method of Horkay and Zrínyi [38] in the small deformation region.

\subsection{Kinetics of temperature induced phase transition}

Prior to the measurements disks of $13 \mathrm{~mm}$ diameter were cut from the swollen film and kept at $20 \pm 0.2{ }^{\circ} \mathrm{C}$ for 2 days to allow them to reach equilibrium. The samples were then plunged into a water bath at $50 \pm 1{ }^{\circ} \mathrm{C}$. The shrinkage induced by the thermal shock was recorded photographically by monitoring the diameter $D$ of the disks. $D$ values are given as the average of five different measurements read from the images by JMicroVision software and compared to the initial diameter $D_{0}$ of the fully swollen gels. 


\subsection{IR sensitivity}

The surface of $10 \times 10 \mathrm{~mm}$ swollen gel films, placed on a glass plate, was exposed to a $\mathrm{CO}_{2}$ laser beam (wavelength: $10.6 \mu \mathrm{m}$, power: $0.500 \mathrm{~W}$, spotsize: $15 \mathrm{~mm}$ ). Thermal maps were recorded at ambient conditions as video files by a Testo 890 thermal imaging camera (Testo, Alton, UK) for 30 seconds prior to the irradiation, then during the 2 minute period of laser exposure. After irradiation the cooling of the gels was monitored for 2.5 minutes. During the irradiation the gel samples collapsed in a circular zone of $5 \mathrm{~mm}$ around the centre of the incident beam. The temperature of the irradiated gels was determined as the average temperature of 20 positions within this zone.

\section{Results and discussion}

\subsection{Characterization}

When CNPs were incorporated the transparent PNIPA gel became either brown (with GO), or black (with CNT). In CNT@PNIPA gels macroscopic heterogeneity due to aggregation can be observed (Figure 3). A 'wall region' and an 'inner region' are clearly distinguishable in the images (not shown here). Typical SEM images of both regions are displayed in Figure 4. In pure PNIPA films these two regions are very similar: wide amorphous pores separated by thin polymer walls. In the composite samples the morphology depends on the type of CNP. Both with CNT and GO the wall and inner regions, respectively, are significantly different, indicating that the orientation of the CNPs might be influenced by interaction with the wall. Nevertheless, the heat transfer during the freezing process may also lead to layer formation. In the composite gel cylinders further morphological differ- ences developed along the axis, as a consequence of sedimentation during gelation, particularly with CNT. The pore size distributions of the CNT containing composites are not very different from pure PNIPA. By contrast, the influence on the pore structure of the GO content is unmistakably stronger (Figure 5). Interestingly, the effect at lower GO content is more marked.

On comparing the swelling degree and the elastic modulus of the hydrogels significant differences appear between the two sets of composites (Figure 6). CNT reduces the swelling degree slightly but monotonically, possibly in part due to the loss of volume occupied by the carbon. CNT has practically no effect on the elastic modulus of these gels.

A more pronounced, non-linear, effect is observed in the $\mathrm{GO}$ composites. Even $15 \mathrm{mg} \mathrm{GO} / \mathrm{g}_{\mathrm{NIPA}}$ content caused an abrupt drop by ca. $33 \%$ in the swelling degree. Further increase of the GO content has no observable effect on the swelling (Figure 6a). Such behaviour arises when the concentration of filler particles reaches the percolation threshold. The elastic modulus increases monotonically in the whole concentration range examined (Figure 6b). A similar observation has been reported by Fan et al. [39] for sodium alginate/polyacrylamide (PAM) hydrogels with comparable GO content $(0.5-5 \mathrm{w} / \mathrm{w} \%)$. PNIPA systems with significantly higher cross-link ratio and 1-10 w/w $\%$ GO content exhibit a contrary trend [34]. Figure 6c illustrates the influence of water content on the modulus. Incorporation of the CNPs substantially improves the compressive strength of the gels (Figure 6d).CNT@NIPA gels tolerate greater deformation before they break, while GO@PNIPA gels withstand higher stress at smaller deformation. The
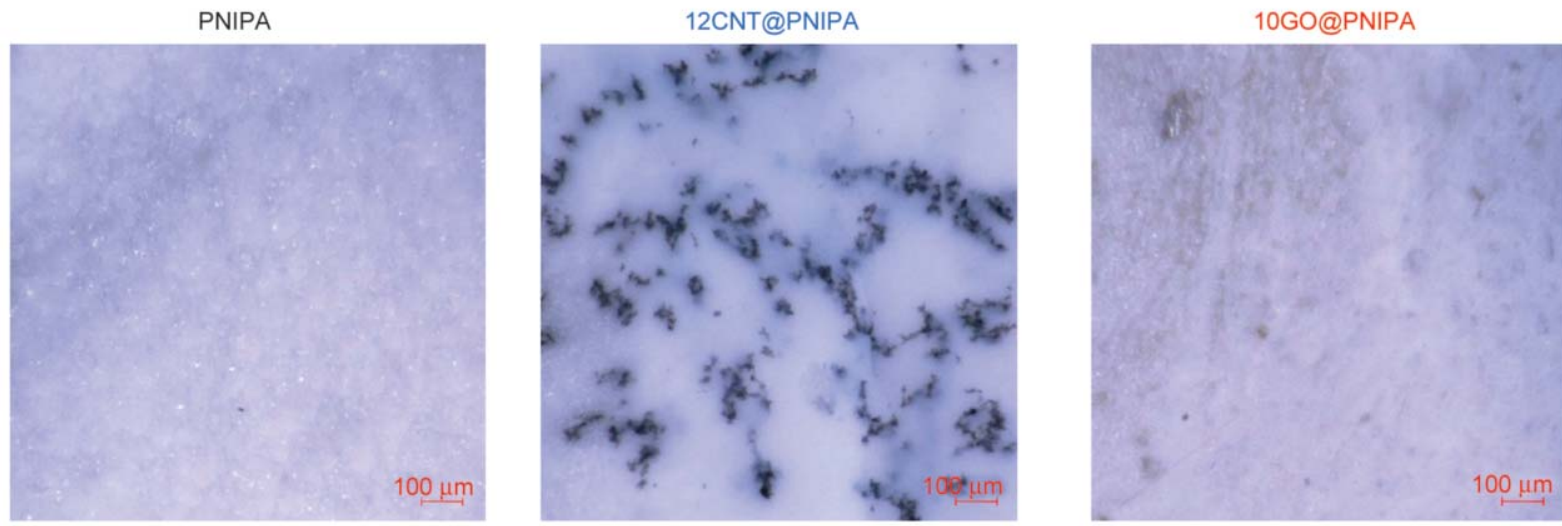

Figure 3. Digital optical microscopic images of the lyophilized cross section of the lyophilised gel films 
Wall region
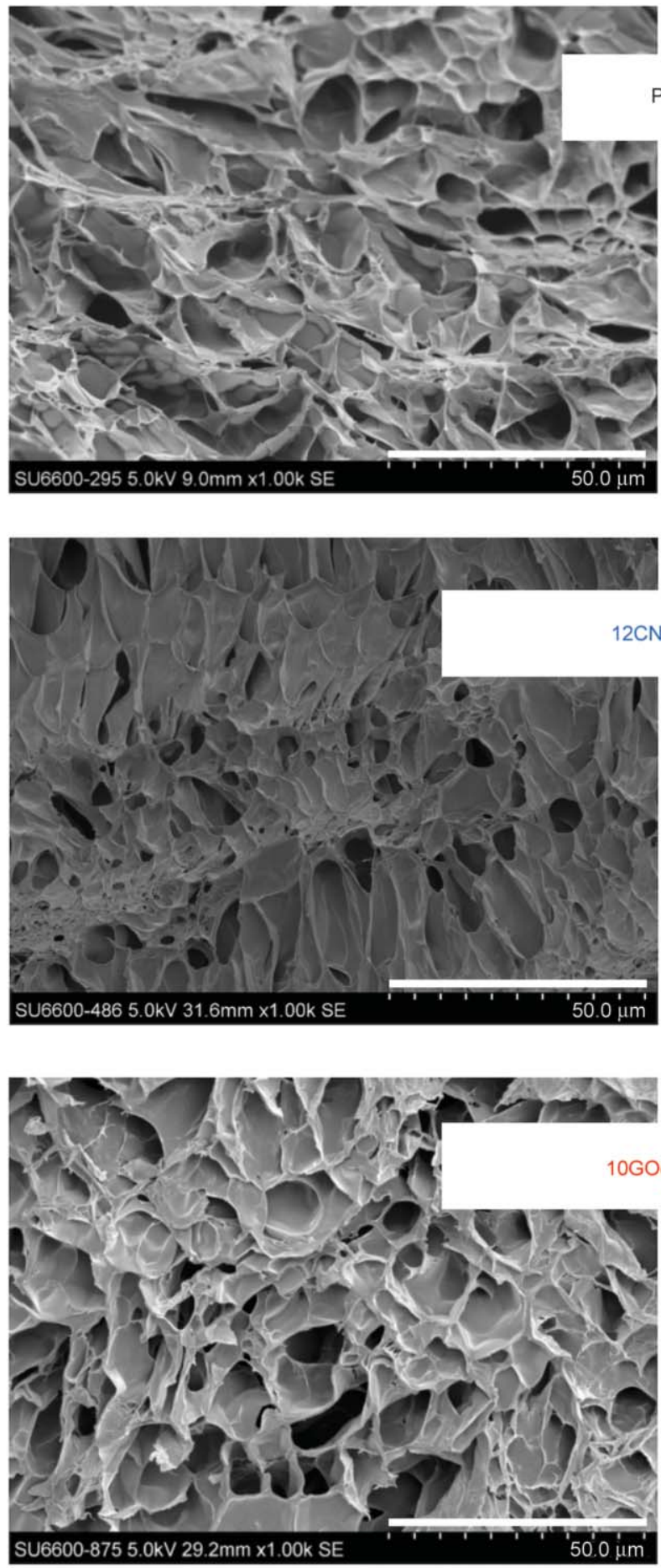

Inner region
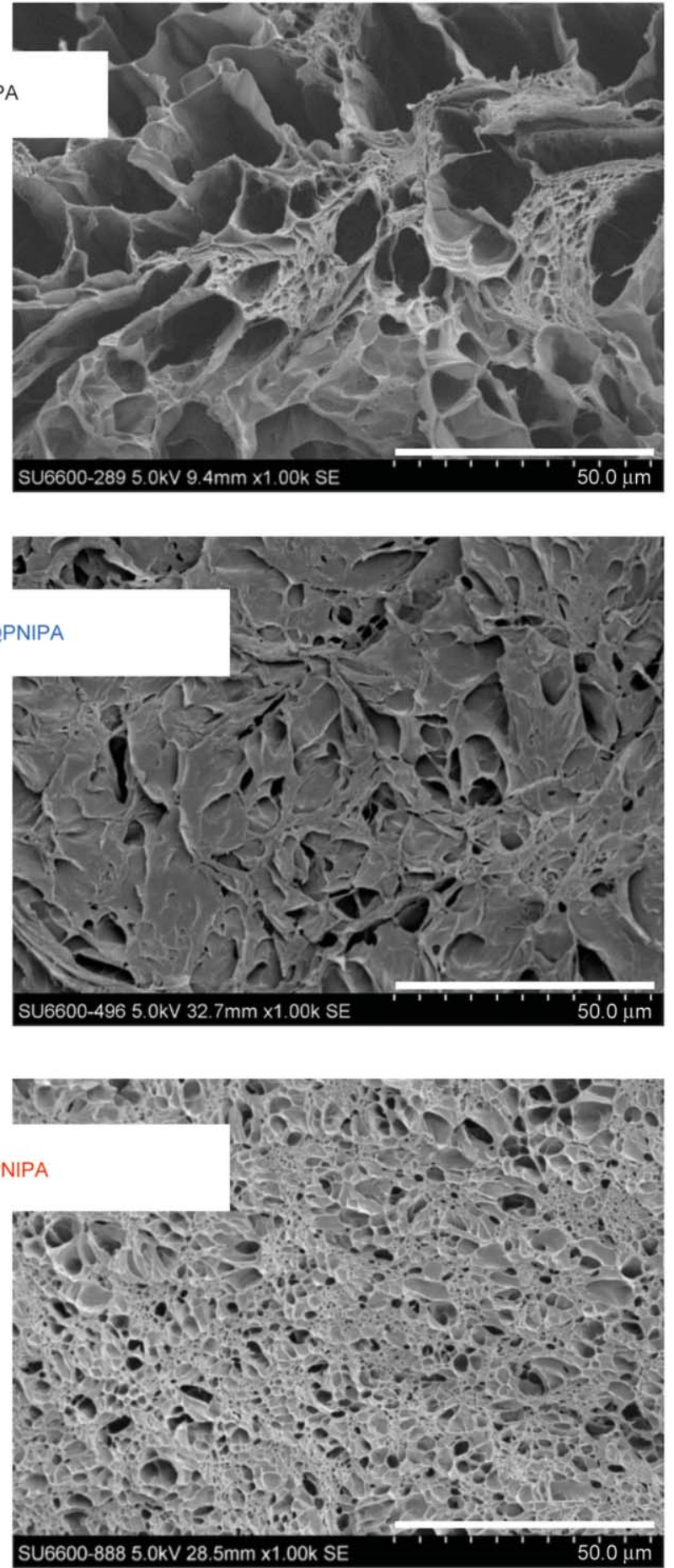

Figure 4. SEM images of CNP@PNIPA gel films dried in cryogenic conditions. Scale bar $50 \mu \mathrm{m}$.

effect of loading on the compressive strength with both fillers is plotted in Figure 6e. When GO is incorporated the compressive strength in PNIPA gels increases by almost an order of magnitude, and is independent of GO content; with CNT@NIPA gels the enhancement is slightly smaller.

\subsection{Deswelling kinetics}

On immersion in warm water disks of CNT@NIPA and GO@NIPA samples immediately turn white, similarly to pure PNIPA. Figure 7 shows that the presence of CNPs appreciably modifies the kinetic response of the PNIPA gel. During the temperature- 

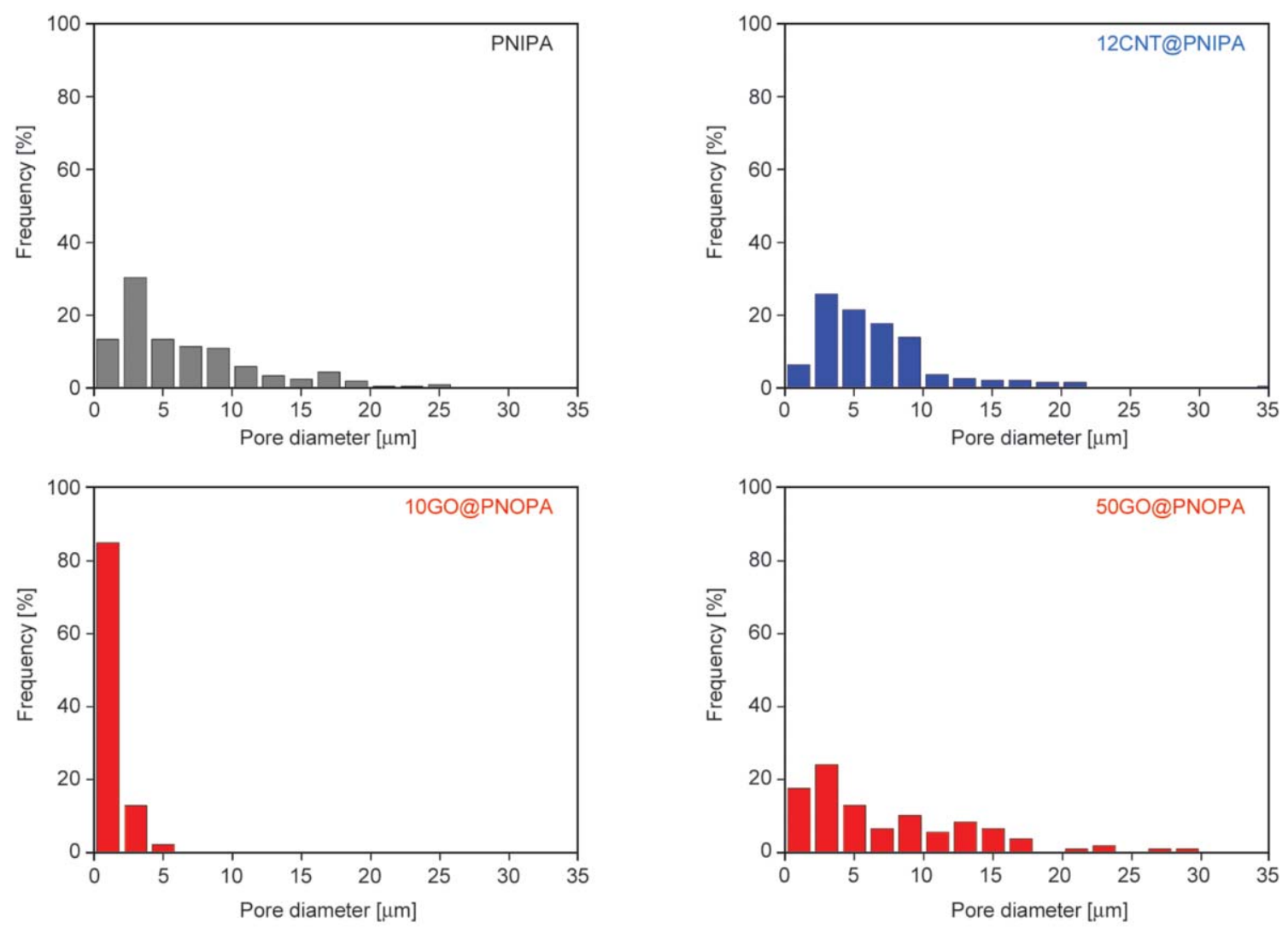

Figure 5. Pore size distribution of the bulk of CNP@PNIPA gel films dried in cryogenic condition
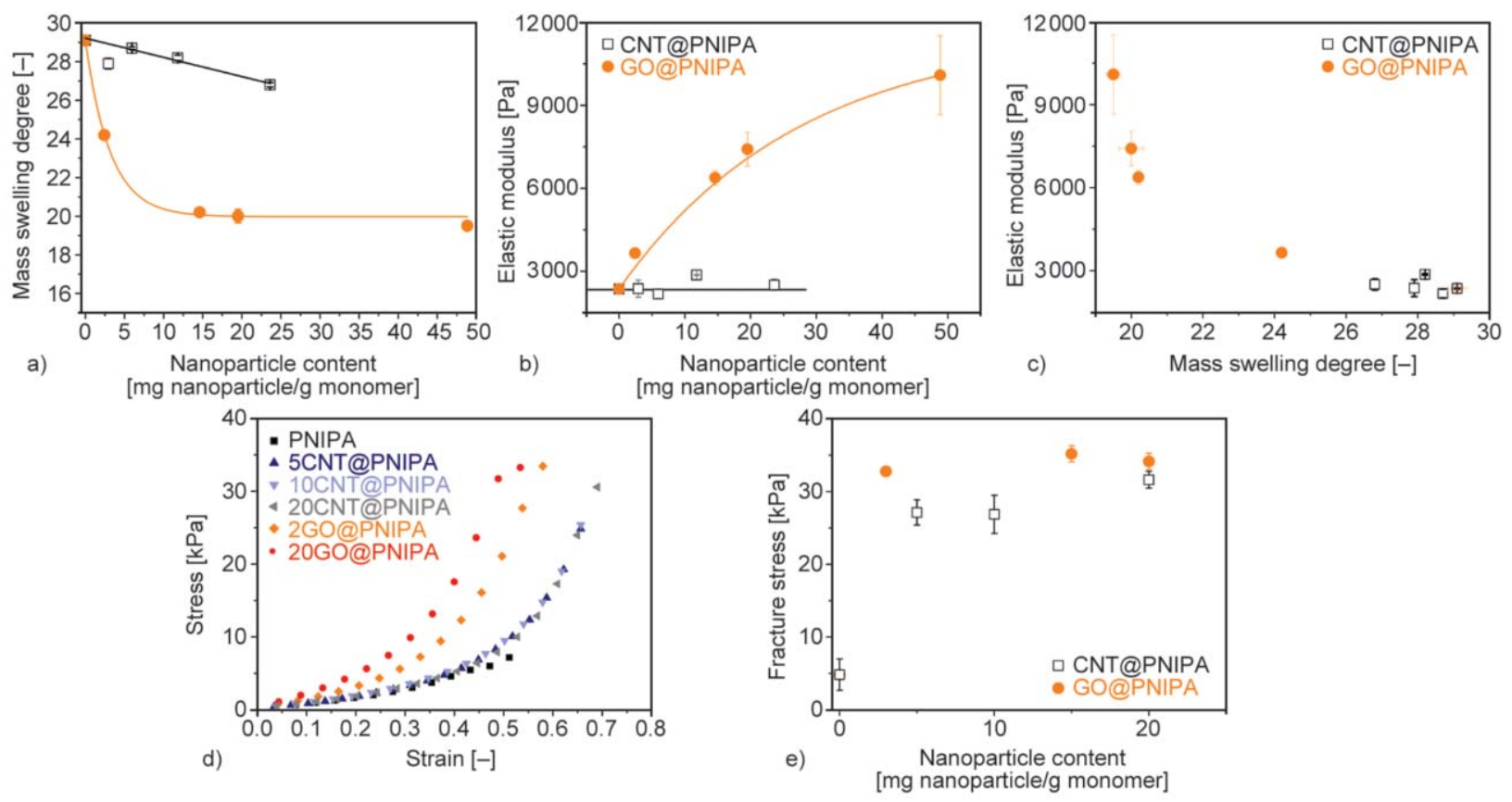

Figure 6. Mass swelling degree in pure water at $20^{\circ} \mathrm{C}$ (a) and elastic modulus (b) of composite gels. Solid lines are guides for the eye. Correlation of modulus and swelling degree (c). Stress-strain curves (d). Compressive strength of composite gels (e).

jump the behaviour of the composite gels is strongly affected by both the quality and quantity of the CNPs. To quantify the effects the experimental shrinkage- curves were fit to a modified exponential decay function shown in Equation (1):

$$
\frac{D}{D_{0}}=\left(\frac{D}{D_{0}}\right)_{\text {fin }}+A_{1} \mathrm{e}^{-\left(\frac{t}{\tau}\right)^{p}}
$$



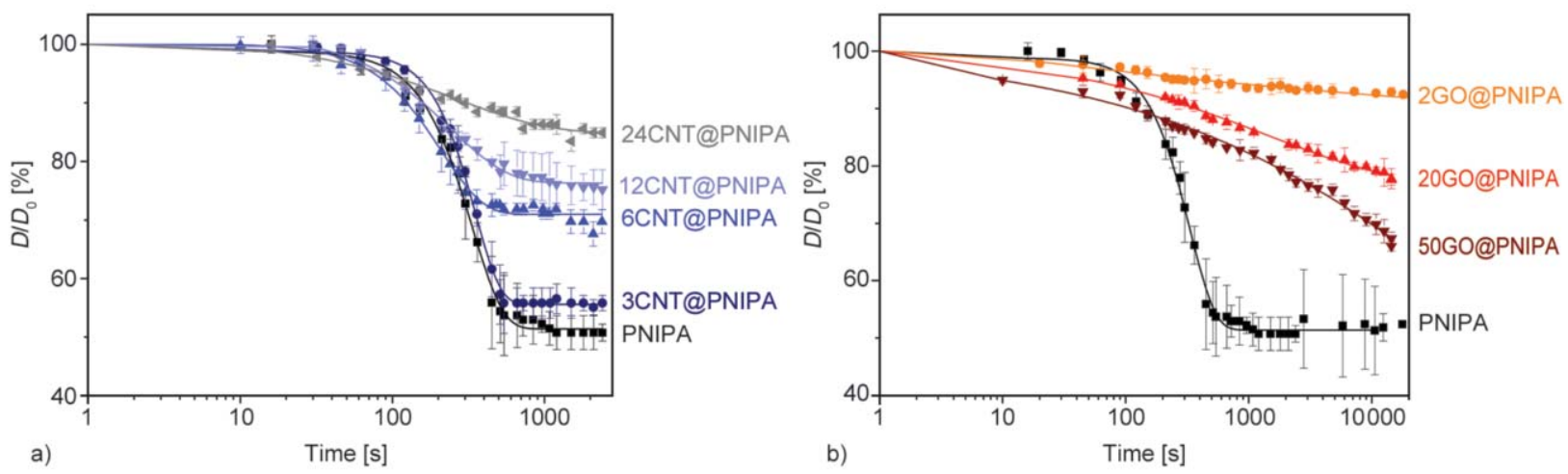

Figure 7. Deswelling kinetics of CNT@PNIPA (a) and GO@PNIPA (b) at $50^{\circ} \mathrm{C}$. Note the order of magnitude difference in range of the $x$-axis scales. Symbols are experimental data, continuous lines are fits to Equation (1).

where $\left(D / D_{0}\right)_{\text {fin }}$ is the final relative diameter, $A$ is a pre-exponential constant and $\tau$ is the time constant of the overall volumetric thermal response. The results are listed in Table 1. The shrinkage curve of the pure PNIPA could be fit by a compressed exponential function $(p>1)$, indicating possible jamming behaviour, with a time constant in good agreement with previous observations [40]. The time constants of the CNT@PNIPA systems are shorter, i.e., CNT slightly accelerates the response of the pure PNIPA gel [40]. $\left(D / D_{0}\right)_{\text {fin }}$ correlates with the CNT content: higher loading results in more limited shrinkage. The value of exponent $p$ decreases systematically and significantly with increasing CNT loading, a sign that deswelling becomes more complex at higher CNT concentrations (Table 1). In 24CNT@PNIPA (the highest CNT content tested) stretched exponential behaviour $(p>1)$ was found, characteristic of multiple relaxation processes with different timescales.

Whereas $2000 \mathrm{~s}$ was largely sufficient for the relaxation of CNT@PNIPA composites, a timeframe of even an order of magnitude longer was insufficient for

Table 1. Fitting parameters of the temperature induced phase transition from Equation (1)

\begin{tabular}{|l|r|r|r|r|c|}
\hline \multicolumn{1}{|c|}{ Sample } & $\begin{array}{c}\left(\frac{\boldsymbol{D}}{\boldsymbol{D}_{\mathbf{0}}}\right)_{\text {fin }} \\
{[\mathbf{\%}]}\end{array}$ & $\begin{array}{c}\boldsymbol{A} \\
{[\%]}\end{array}$ & $\begin{array}{c}\boldsymbol{\tau} \\
{[\mathbf{s}]}\end{array}$ & $\boldsymbol{p}$ & $\boldsymbol{R}^{\mathbf{2}}$ \\
\hline PNIPA & 51.4 & 47.5 & 334 & 2.14 & 0.995 \\
\hline 3CNT@PNIPA & 55.6 & 43.2 & 348 & 2.58 & 0.997 \\
\hline 6CNT@PNIPA & 71.0 & 29.1 & 211 & 1.60 & 0.990 \\
\hline 12CNT@PNIPA & 76.3 & 25.4 & 241 & 1.15 & 0.987 \\
\hline 24CNT@PNIPA & 84.9 & 16.0 & 259 & 0.721 & 0.971 \\
\hline 2GO@PNIPA & 90.5 & 9.5 & 1437 & 0.266 & 0.932 \\
\hline 20GO@PNIPA & 76.5 & 23.7 & 1470 & 0.412 & 0.994 \\
\hline 50GO@PNIPA & 34.2 & 60.1 & 53762 & 0.286 & 0.992 \\
\hline
\end{tabular}

$\left(D / D_{0}\right)_{\text {fin }}$ : relative diameter, $A$ : pre-exponential factor; $\tau$ : time constant of deswelling response; $p$ : exponent; $R^{2}$ : coefficient of determination the GO@PNIPA gels (Figure 7). For the GO@PNIPA samples the values of the fitting parameters (Table 1) can be used only for qualitative comparisons, as the absence of a measured asymptotic value of $\left(D / D_{0}\right)_{\text {fin }}$ makes the fitting parameters uncertain. It can nevertheless be concluded that, unlike CNT, the lowest GO content has the strongest effect on deswelling kinetics. Substantial slowing down of the thermal response is observed at all three concentrations (Figure 8), which resembles the effect of increased cross-linking density in pure PNIPA gels [40]. All curve fits yielded a stretching parameter $p<1$, indicating multiple processes.

\subsection{IR sensitivity}

Recent experimental and theoretical studies found that the presence of network structure increases thermal diffusivity in PNIPA gels compared to pure water. It was also observed that latent heat influences thermal diffusivity [41].

Carbon materials including nanoparticles are known for their high IR absorption. As expected [25-27],

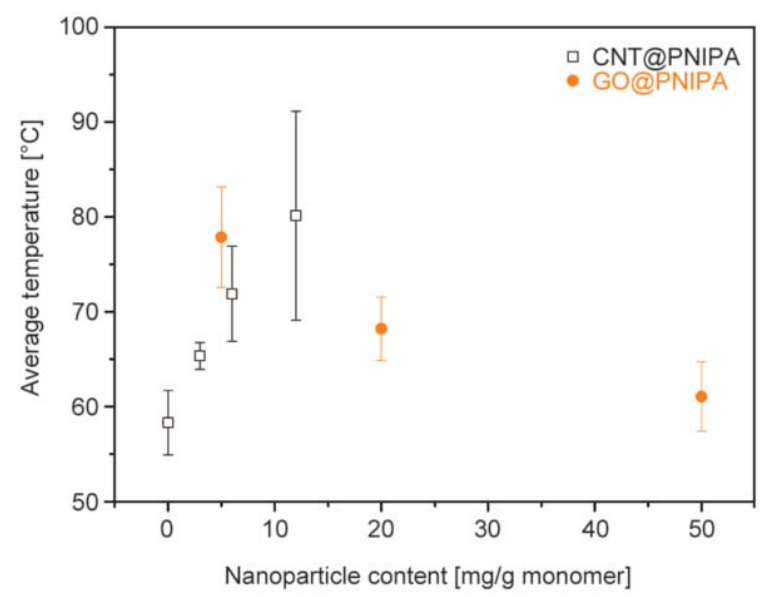

Figure 8. Average temperature values of PNIPA composites during IR laser exposure 

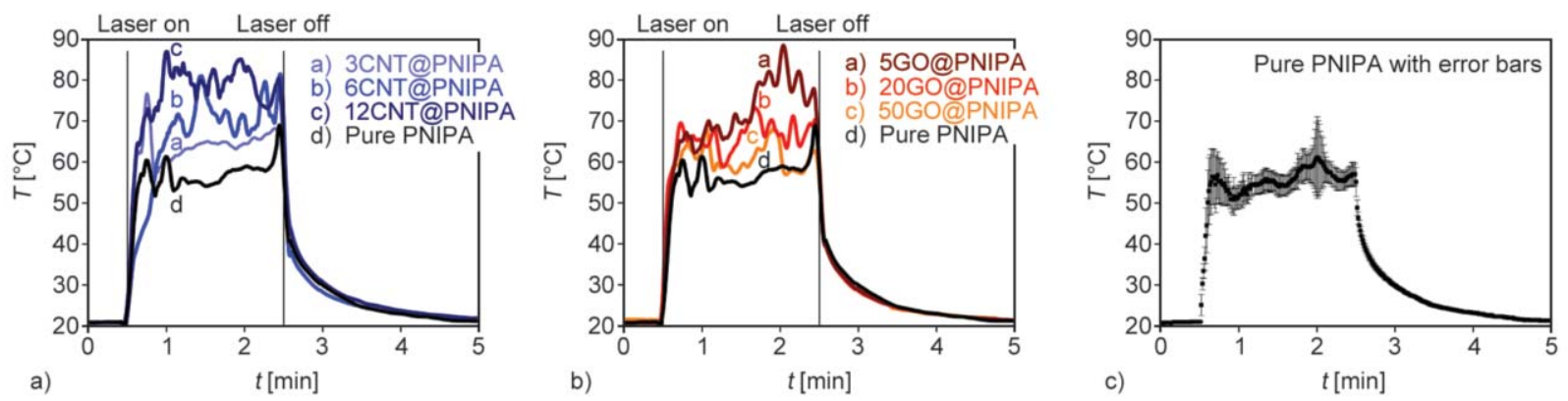

Figure 9. Temperature $(T)$ profile of PNIPA composites upon IR laser exposure $\left(t\right.$ : observation time) at $20^{\circ} \mathrm{C}(\mathrm{a}, \mathrm{b})$, temperature profile of pure PNIPA with error bars (c)

addition of both CNT and GO results in enhanced IR sensitivity (Figures 8 and 9). The doped systems, however, display significant differences according to the type and concentration of nanoparticle incorporated. Fast shrinkage on exposure to IR laser irradiation, and quick recovery of the gels after exposure is observed in all cases. During the exposure the gel samples collapse in a circular zone of diameter $5 \mathrm{~mm}$ centred around the incident beam. The measured temperature fluctuations may be attributed to the low thermal conductivity of the gel, even in the presence of CNPs (Figure 9a and Figure 9b). In both sets of systems a monotonic correlation was found between the nanoparticle content and the temperature of the sample, but with different trend. In the CNT@PNIPA systems the temperature rise in the gels with increasing CNT concentration was proportionally higher (Figure 8). Incorporating a small amount of GO into PNIPA resulted in the same enhanced response as CNT@NIPA with a similar CNT content, but further addition of GO systematically reduced the effect.

\subsection{Discussion}

The differences observed between the effects of the two different types of CNPs may stem more from their chemical behaviour than their geometrical shape. Multiwall carbon nanotubes contain Russian dolllike concentric tubes consisting of graphite like carbon arrays. The outermost cylinder is decorated with O-containing surface functional groups, but their concentration is relatively low. Therefore the functional groups do not significantly modify the aromatic graphitic structure. That is, during the PNIPA synthesis, the reactivity of the CNTs present in the radical polymerization is modest. However, as their surface is predominantly hydrophobic, they tend to aggregate, even after intense ultrasonication. GO, by contrast, is composed of a few strongly damaged graphene sheets richly decorated with $\mathrm{O}$. The delocalised electron system is therefore severely disrupted and most of the electrons are localised as $\mathrm{C}=\mathrm{C}$ double bonds, which are reactive in radical reactions. The hydrophilic surface also ensures good dispersibility. Owing to their surface chemistry, the GO platelets are well distributed in the polymerisation medium, which allows them to form a percolating GO network, consisting of hubs that are each covalently linked to several polymer chains; this outcome is less probable with the CNT aggregates. This difference in behaviour affects the response of the loaded gels during the swelling and stress-strain observations. While GO units build up to form an interpenetrating network that is strongly connected to the polymer chains, the CNTs act as 'free' aggregates. Increasing the CNT content increases the number of aggregates in the precursor solution. Hydrophilic GO forms bulky, randomly oriented GO clusters only at high concentrations.

The difference in electron structure also leads to the different heat conductivity and IR absorption performance of the gels when the two CNPs are present. At low concentration CNT has only a limited influence on the deswelling kinetics. Although increasing the CNT content may improve the heat conductivity, higher amounts of CNT filler delay the relaxation and increase the time constant. The chains must relax in the vicinity of more and more aggregates. Also, with CNTs, the higher their amount in the gel the greater is their IR absorption.

In the GO@PNIPA systems, the complexity of the deswelling process is reflected by the $p<1$ relation that appears already at the lowest concentration. Several process may superimpose: i) increasing the GO units increases the number of potentially reactive 
double bonds with no increase in the NIPA and BA concentration; ii) at a certain threshold concentration the GO platelets percolates and forms a connected interpenetrating network; iii) overall gel collapse is a slow process that requires ordering or stacking of the platelets. The strong IR absorption at low GO concentration and weaker absorption at high concentration than with CNT is further evidence of its connected network structure. In spite of the lower intrinsic thermal conductivity of GO, its percolating network inside the gel conducts heat more efficiently from the polymer matrix into the substrate than do the isolated CNT clusters [42].

\section{Conclusions}

Incorporation of CNT and GO into PNIPA hydrogels produces different effects on the behaviour of the hybrids. Apart from influencing the porous morphology of the composites, their effect on the swelling degree and the elastic modulus shows a different trend. The elastic modulus of PNIPA gel is enhanced by GO, while the swelling degree of the GO@PNIPA systems decreases significantly. By contrast, the swelling and mechanical properties of CNT@PNIPA composites are similar to those of pure PNIPA. Both types of particle substantially enhance the fracture stress tolerance of the PNIPA hydrogel.

Significant differences are observed in the thermal response of the two systems. The time constant and swelling ratio of the temperature-induced shrinkage can therefore be adjusted by selecting the type and amount of nanoparticle loading. This could provide a means for accurately controlling deswelling kinetics, e.g., in the drug release profile of PNIPA systems. This capacity could also be employed in sensor applications, where fast and excessive shrinkage can be a significant drawback. Both CNT and GO enhance the infrared sensitivity of the PNIPA gel, thus opening a route for constructing novel drug transport and actuator systems. The novelty of the present findings is to show that the influence on the gel behaviour of CNT and GO is different. This difference stems both from their different chemical reactivity during the gel synthesis and from their different geometry. In the one case the CNTs aggregate into separate clusters, while in the other the GO disperses in the gel to form a percolating, thermally conducting, network.

\section{Acknowledgements}

The authors thank Prof. K. Kaneko for providing the graphite sample and Prof. T. Maekawa for his valuable advice in the IR experiments. The financial support of Hungarian grant OTKA K101861 and EU project FP7-PEOPLE-2010-IRSES269267 is acknowledged.

\section{References}

[1] Bradbury J. H., Fenn M. D., Gosney I.: The change of volume associated with the helix-coil transition in poly$\gamma$-benzyl-L-glutamate. Journal of Molecular Biology, 11, 137-140 (1965). DOI: $10.1016 / \mathrm{S} 0022-2836(65) 80179-6$

[2] Heskins M., Guillet J. E.: Solution properties of poly ( $N$-isopropylacrylamide). Journal of Macromolecular Science Part A: Chemistry, 2, 1441-1455 (1968). DOI: $10.1080 / 10601326808051910$

[3] Tanaka T.: Collapse of gels and the critical endpoint. Physical Review Letters, 40, 820-823 (1978). DOI: 10.1103/PhysRevLett.40.820

[4] Ricka J., Tanaka T.: Swelling of ionic gels: Quantitative performance of the Donnan theory. Macromolecules, 17, 2916-2921 (1984). DOI: $10.1021 / \mathrm{ma} 00142 \mathrm{a} 081$

[5] Bae Y. H., Okano T., Kim S. W.: Temperature dependence of swelling of crosslinked poly $\left(N, N^{\prime}\right.$-alkyl substituted acrylamides) in water. Journal of Polymer Science Part B: Polymer Physics, 28, 923-936 (1990). DOI: 10.1002/polb.1990.090280609

[6] Reinicke S., Döhler S., Tea S., Krekhova M., Messing R., Schmidt A. M., Schmalz H.: Magneto-responsive hydrogels based on maghemite/triblock terpolymer hybrid micelles. Soft Matter, 6, 2760-2773 (2010).

DOI: $10.1039 / \mathrm{c} 000943 \mathrm{a}$

[7] Schmaljohann D.: Thermo- and pH-responsive polymers in drug delivery. Advanced Drug Delivery Reviews, 58, 1655-1670 (2006).

DOI: 10.1016/j.addr.2006.09.020

[8] Manek E., Domján A., Madarász J., László K.: Interactions in aromatic probe molecule loaded poly $(N$-isopropylacrylamide) hydrogels and implications for drug delivery. European Polymer Journal, 68, 657-664 (2015). DOI: $10.1016 /$ j.eurpolymj.2015.03.043

[9] Kumar A., Srivastava A., Galaev I. Y., Mattiasson B.: Smart polymers: Physical forms and bioengineering applications. Progress in Polymer Science, 32, 1205-1237 (2007).

DOI: 10.1016/j.progpolymsci.2007.05.003

[10] Ionov L.: Hydrogel-based actuators: Possibilities and limitations. Materials Today, 17, 494-503 (2014). DOI: 10.1016/j.mattod.2014.07.002 
[11] Depa K., Strachota A., Šlouf M., Hromádková J.: Fast temperature-responsive nanocomposite PNIPAM hydrogels with controlled pore wall thickness: Force and rate of T-response. European Polymer Journal, 48, 1997 2007 (2012).

DOI: 10.1016/j.eurpolymj.2012.09.007

[12] Coughlan D. C., Corrigan O. I.: Drug-polymer interactions and their effect on thermoresponsive poly $(\mathrm{N}$-isopropylacrylamide) drug delivery systems. International Journal of Pharmaceutics, 313, 163-174 (2006). DOI: $10.1016 /$ j.ijpharm.2006.02.005

[13] Schexnailder P., Schmidt G.: Nanocomposite polymer hydrogels. Colloid and Polymer Science, 287, 1-11 (2009).

DOI: $10.1007 / \mathrm{s} 00396-008-1949-0$

[14] Cirillo G., Hampel S., Spizzirri U. G., Parisi O. I., Picci N., Iemma F.: Carbon nanotubes hybrid hydrogels in drug delivery: A perspective review. BioMed Research International, 2014, 825017/1-825017/17 (2014). DOI: 10.1155/2014/825017

[15] Sahoo N. G., Rana S., Cho J. W., Li L., Chan S. H.: Polymer nanocomposites based on functionalized carbon nanotubes. Progress in Polymer Science, 35, 837-867 (2010).

DOI: 10.1016/j.progpolymsci.2010.03.002

[16] Pandey G., Wolters M., Thostenson E. T., Heider D.: Localized functionally modified glass fibers with carbon nanotube networks for crack sensing in composites using time domain reflectometry. Carbon, 50, 38163825 (2012).

DOI: $10.1016 /$ j.carbon.2012.04.008

[17] Tsonos C., Pandis C., Soin N., Sakellari D., Myrovali E., Kripotou S., Kanapitsas A., Siores E.: Multifunctional nanocomposites of poly(vinylidene fluoride) reinforced by carbon nanotubes and magnetite nanoparticles. Express Polymer Letters, 9, 1104-1118 (2015).

DOI: $10.3144 /$ expresspolymlett.2015.99

[18] Voitko K., Tóth A., Demianenko E., Dobos G., Berke B., Bakalinska O., Grebenyuk A., Tombácz E., Kuts V., Tarasenko Y., Kartel M., László K.: Catalytic performance of carbon nanotubes in $\mathrm{H}_{2} \mathrm{O}_{2}$ decomposition: Experimental and quantum chemical study. Journal of Colloid and Interface Science, 437, 283-290 (2015).

DOI: $10.1016 /$ j.jcis.2014.09.045

[19] Tóth A., Törőcsik A., Tombácz E., Oláh E., Heggen M., Li C., Klumpp E., Geissler E., László K.: Interaction of phenol and dopamine with commercial MWCNTs. Journal of Colloid and Interface Science, 364, 469-475 (2011). DOI: $10.1016 /$ j.jcis.2011.08.044

[20] Yun J., Im J. S., Lee Y-S., Kim H-I.: Electro-responsive transdermal drug delivery behavior of PVA/PAA/ MWCNT nanofibers. European Polymer Journal, 47, 1893-1902 (2011).

DOI: $10.1016 /$ j.eurpolymj.2011.07.024
[21] Kim H., Abdala A. A., Macosko C. W.: Graphene/polymer nanocomposites. Macromolecules, 43, 6515-6530 (2010).

DOI: $10.1021 / \mathrm{ma} 100572 \mathrm{e}$

[22] Kuila T., Bose S., Mishra A. K., Khanra P., Kim N. H., Lee J. H.: Chemical functionalization of graphene and its applications. Progress in Materials Science, 57, 10611105 (2012).

DOI: $10.1016 /$ j.pmatsci.2012.03.002

[23] Wu X., Lin T. F., Tang Z. H., Guo B. C., Huang G. S.: Natural rubber/graphene oxide composites: Effect of sheet size on mechanical properties and straininduced crystallization behavior. Express Polymer Letters, 9, 672-685 (2015).

DOI: 10.3144/expresspolymlett.2015.63

[24] Pedrazzoli D., Dorigato A., Conti T., Vanzetti L., Bersani M., Pegoretti A.: Liquid crystalline polymer nanocomposites reinforced with in-situ reduced graphene oxide. Express Polymer Letters, 9, 709-720 (2015).

DOI: 10.3144/expresspolymlett.2015.66

[25] Zhu C-H., Lu Y., Peng J., Chen J-F., Yu S-H.: Photothermally sensitive poly( $N$-isopropylacrylamide $) /$ graphene oxide nanocomposite hydrogels as remote light-controlled liquid microvalves. Advanced Functional Materials, 22, 4017-4022 (2012).

DOI: $10.1002 / \mathrm{adfm} .201201020$

[26] Miyako E., Nagata H., Hirano K., Hirotsu T.: Photodynamic thermoresponsive nanocarbon-polymer gel hybrids. Small, 4, 1711-1715 (2008).

DOI: $10.1002 / \mathrm{smll} .200800601$

[27] Zhang X., Pint C. L., Lee M. H., Schubert B. E., Jamshidi A., Takei K., Ko H., Gillies A., Bardhan R., Urban J. J., Wu M., Fearing R., Javey A.: Optically- and thermally-responsive programmable materials based on carbon nanotube-hydrogel polymer composites. Nano Letters, 11, 3239-3244 (2011). DOI: $10.1021 / \mathrm{nl} 201503 \mathrm{e}$

[28] Szeluga U., Kumanek B., Trzebicka B.: Synergy in hybrid polymer/nanocarbon composites. A review. Composites Part A: Applied Science and Manufacturing, 73, 204-231 (2015).

DOI: $10.1016 /$ j.compositesa.2015.02.021

[29] Chen Y-S., Tsou P-C., Lo J-M., Tsai H-C., Wang Y-Z., Hsiue G-H.: Poly( $N$-isopropylacrylamide) hydrogels with interpenetrating multiwalled carbon nanotubes for cell sheet engineering. Biomaterials, 34, 7328-7334 (2013).

DOI: $10.1016 /$ j.biomaterials.2013.06.017

[30] Islam M. F., Alsayed A. M., Dogic Z., Zhang J., Lubensky T. C., Yodh A. G.: Nematic nanotube gels. Physical Review Letters, 92, 088303/1-088303/4 (2004). DOI: $10.1103 /$ PhysRevLett.92.088303

[31] Yang Z., Cao Z., Sun H., Li Y.: Composite films based on aligned carbon nanotube arrays and a poly $(N$-isopropyl acrylamide) hydrogel. Advanced Materials, 20, 2201-2205 (2008).

DOI: $10.1002 / \mathrm{adma} .200701964$ 
[32] Li Z., Shen J., Ma H., Lu X., Shi M., Li N., Ye M.: Preparation and characterization of $\mathrm{pH}$ - and temperature-responsive hydrogels with surface-functionalized graphene oxide as the crosslinker. Soft Matter, 8, 31393145 (2012).

DOI: $10.1039 / \mathrm{c} 2 \mathrm{sm} 07012 \mathrm{j}$

[33] Lo C-W., Zhu D., Jiang H.: An infrared-light responsive graphene-oxide incorporated poly $(N$-isopropylacrylamide) hydrogel nanocomposite. Soft Matter, 7, 5604 5609 (2011).

DOI: $10.1039 / \mathrm{c} 1 \mathrm{sm} 00011 \mathrm{j}$

[34] Ma X., Li Y., Wang W., Ji Q., Xia Y.: Temperature-sensitive poly( $N$-isopropylacrylamide)/graphene oxide nanocomposite hydrogels by in situ polymerization with improved swelling capability and mechanical behavior. European Polymer Journal, 49, 389-396 (2013). DOI: 10.1016/j.eurpolymj.2012.10.034

[35] Wang Z., Shirley M. D., Meikle S. T., Whitby R. L. D., Mikhalovsky S. V.: The surface acidity of acid oxidised multi-walled carbon nanotubes and the influence of insitu generated fulvic acids on their stability in aqueous dispersions. Carbon, 47, 73-79 (2009).

DOI: $10.1016 /$ j.carbon.2008.09.038

[36] Marcano D. C., Kosynkin D. V., Berlin J. M., Sinitskii A., Sun Z., Slesarev A., Alemany L. B., Lu W., Tour J. M.: Improved synthesis of graphene oxide. ACS Nano, 4, 4806-4814 (2010).

DOI: $10.1021 / \mathrm{nn} 1006368$

[37] László K., Kosik K., Rochas C., Geissler E.: Phase transition in poly $(N$-isopropylacrylamide) hydrogels induced by phenols. Macromolecules, 36, 7771-7776 (2003). DOI: $10.1021 / \mathrm{ma} 034531 \mathrm{u}$
[38] Horkay F., Zrínyi M.: Studies on the mechanical and swelling behavior of polymer networks based on the scaling concept. 4. Extension of the scaling approach to gels swollen to equilibrium in a diluent of arbitrary activity. Macromolecules, 15, 1306-1310 (1982). DOI: 10.1021/ma00233a018

[39] Fan J., Shi Z., Lian M., Li H., Yin J.: Mechanically strong graphene oxide/sodium alginate/polyacrylamide nanocomposite hydrogel with improved dye adsorption capacity. Journal of Materials Chemistry A, 1, 7433-7443 (2013).

DOI: $10.1039 / \mathrm{c} 3 \operatorname{ta} 10639 \mathrm{j}$

[40] László K., Fluerasu A., Moussaïd A., Geissler E.: Deswelling kinetics of PNIPA gels. Soft Matter, 6, 4335-4338 (2010). DOI: $10.1039 /$ C0SM00297F

[41] Tél A., Bauer R. A., Varga Z., Zrínyi M.: Heat conduction in poly $(N$-isopropylacrylamide $)$ hydrogels. International Journal of Thermal Sciences, 85, 47-53 (2014). DOI: 10.1016/j.ijthermalsci.2014.06.005

[42] Karásek L., Meissner B., Asai S., Sumita M.: Percolation concept: Polymer-filler gel formation, electrical conductivity and dynamic electrical properties of carbon-black-filled rubbers. Polymer Journal, 28, 121-126 (1996). DOI: 10.1295/polymj.28.121 